\title{
Point-By-Point Inscription of Sub-Micrometer Period Fiber Bragg Gratings
}

\author{
Y. Lai, K. Zhou, K. Sugden, I. Bennion \\ Photonics Research Group, School of Engineering and Applied Science, Aston University, Birmingham B4 7ET, United Kingdom \\ email: lai_yicheng@dsi.a-star.edu.sg
}

\begin{abstract}
Point-by-point inscription of sub- $\mu$ m period fiber Bragg gratings with good spectral quality, $1^{\text {st }}$ order Bragg resonances within the C-band is achieved. Distinct polarization characteristics are further observed in these fiber gratings.

(C)2008 Optical Society of America

OCIS codes: (320.2250) Femtosecond phenomena; (060.3735) Fiber Bragg gratings; (260.1440) Birefringence
\end{abstract}

\section{Introduction}

Point-by-point (PBP) grating inscription using focused femtosecond laser pulses is an attractive fiber Bragg grating (FBG) fabrication technique since it eliminates the need of a phase mask and Bragg resonances can be achieved at various useful wavelengths with ease. To date, such direct inscription technique has largely rely on higher order grating structures in order to achieve strong, quality spectral resonances at the desired wavelengths [e.g. 1,2]. For a fixed inscribed length and index modulation, one can expect the Bragg response from its $1^{\text {st }}$ order grating structure to be more pronounced than that from a grating operating in the higher order since the number of constituent periods is reduced for the latter. However, $1^{\text {st }}$ order FBGs fabricated to date only reveal resonances of modest strength with undesired spectral distortions and on the contrary, the Bragg response from the higher order grating structure outperforms that from its $1^{\text {st }}$ order counterpart [2].

In this paper, we demonstrate PBP inscription of FBGs in standard single-mode fiber with $1^{\text {st }}$ order Bragg resonances within the $\mathrm{C}$-band using an IR femtosecond laser. The inscription conditions to optimize the grating fabrication have been explored to achieve 5mm-long, sub- $\mu \mathrm{m}$ period FBGs with high spectral quality ${ }^{\text {st }}$ order Bragg resonances, using only $75 \mathrm{~nJ}$ inscription pulse energy. Furthermore, the gratings exhibit birefringence and polarization-dependent reflectivity of a magnitude not observed before.

\section{Experiment}

Femtosecond laser pulses of $150 \mathrm{fs}$ pulse width at $800 \mathrm{~nm}$ from an amplified Ti:Sapphire laser system $(1 \mathrm{kHz}$ repetition rate) were focused into the fiber by a $100 \times$ microscopic objective $(\mathrm{NA}=0.55$, working distance $=13 \mathrm{~mm}$ ). The fiber was moved at a constant speed along the fiber axis so that each laser pulse produces a grating pitch in the fiber at the beam focal point. It has been illustrated that the intrinsic fiber geometry can cause significant distortion to an incident focused laser beam [3]. The surface curvature of the fiber introduces a cylindrical lens effect which leads to differential focusing conditions to the orthogonal planes of the incident femtosecond pulse. Such distortion increases as the focal point moves closer towards the fiber core, expanding the pulse energy over a larger focal volume [3]. Periodic index formation is hence hindered by the overlapping of the laser focal volumes, which severely limits the grating order achievable, particularly for closely-spaced periods. To help visualize the distortion effect in a grating inscription process, two grating structures of $3 \mu \mathrm{m}$ grating period were fabricated using the PBP inscription technique into the fiber cladding and the fiber core separately. As shown in Fig. 1a and 1b, comparing the observed inscribed structures, it is evident that as the laser focus is placed closer to the fiber core the grating structure is distorted to the extent that the fringes are no longer visible.
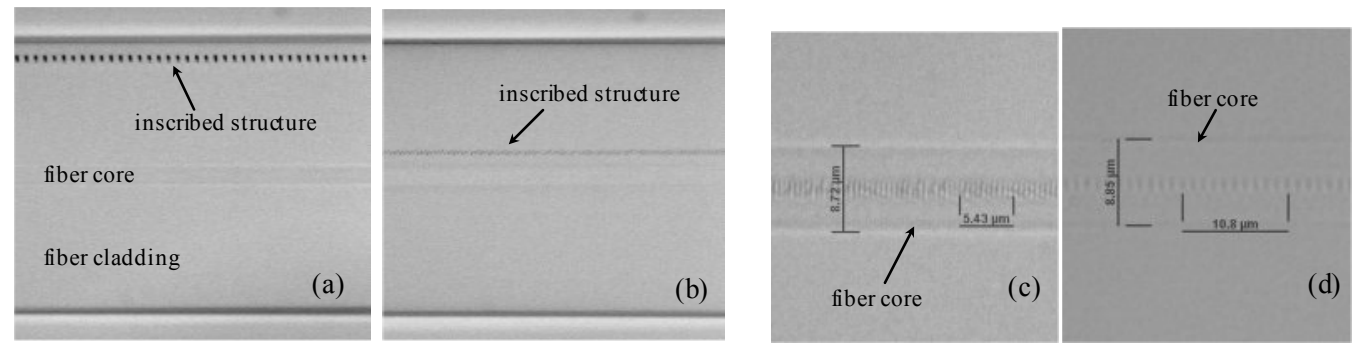

Fig. 1. Microscope images of inscribed gratings in a (a) fiber cladding and, (b) fiber core under identical fabrication conditions without alleviating the distortion effect by the fiber surface on the inscription beam. Resolving the distortion effect enables highly-localized index modifications for inscribing (a) $0.54 \mu \mathrm{m}$ and, (b) $1.08 \mu \mathrm{m}$ period grating structures in the core. 
To overcome the fabrication limitation imposed by the intrinsic fiber geometry, the fiber was placed within a square glass capillary tube filled with index-matching oil so that the surface geometry presented to the path of the incident femtosecond laser is flat [3]. A 5mm-long grating was inscribed into the fiber core using a translation speed of $0.539 \mathrm{~mm} / \mathrm{s}$ with measured pulse energy of $75 \mathrm{~nJ}$. This led to a grating period of $0.539 \mu \mathrm{m}$ with a $1^{\text {st }}$ order Bragg resonance at $\sim 1561 \mathrm{~nm}$. In a separate fiber, a $5 \mathrm{~mm}$-long $2^{\text {nd }}$ order grating structure was similarly inscribed with the same pulse energy using a translation speed of $1.078 \mathrm{~mm} / \mathrm{s}$. The inscribed structures viewed under a $100 \times$ microscope lens are shown in Fig. 1c and 1d respectively.

The inscribed gratings actually consists of an array of individual ellipsoidal index perturbations, each formed by a single laser pulse, with observed width and depth of $\sim 0.3 \mu \mathrm{m}$ and $\sim 1.9 \mu \mathrm{m}$ respectively (aspect ratio of 6.3 ). In line with previous studies [4], these elliptical features are attributed to the longitudinal distribution of the spatial intensity of the beam focused by an objective. Despite the close proximity of the grating pitches, the $0.54 \mu \mathrm{m}$ and $1.08 \mu \mathrm{m}$ index modulation periods are readily visible. The square capillary tube has effectively alleviated the fabrication limitations imposed by the fiber geometry to preserve the tight focusing conditions of the incident pulse.
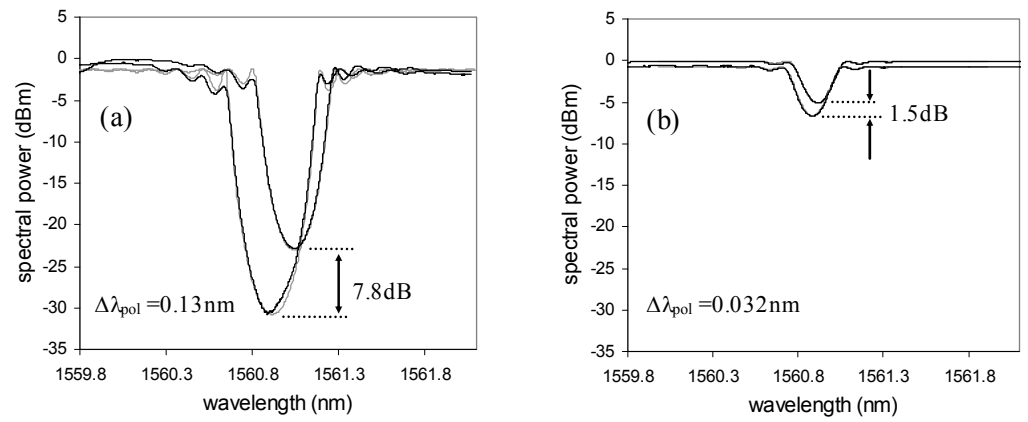

Fig. 2. Superimposed measured and simulated (grey) transmission spectra profiles along orthogonal polarization states of a (a) $1^{\text {st }}$ order FBG and, (b) $2^{\text {nd }}$ order FBG of identical length and fabrication pulse energy. All spectra profiles are scaled identically for comparison.

On the other hand, the asymmetry of the induced index profile with respect to the circular cross-sectional area of the fiber core suggests that the gratings would exhibit polarization-dependent spectral responses. Using a polarized light measurement system, the transmission profiles of the gratings along orthogonal polarization states are measured and the normalized results are fitted to simulations based on the transfer matrix method as shown in Fig. 2. For the $1^{\text {st }}$ order grating structure, the difference in Bragg wavelengths between orthogonal polarization states, denoted as $\Delta \lambda_{\text {pol }}$, measured $0.13 \mathrm{~nm}$, corresponding to an effective birefringence, $\Delta \mathrm{n}_{\text {eff }}$, on the order of $1.2 \times 10^{-4}$. Based on the closely-fitted simulation results, the effective grating strength parameter, $\kappa \mathrm{L}$, along each polarization state are 4.09 and 3.19. This leads to a difference in transmission dips, $\Delta \gamma$, at Bragg wavelengths of 7.8dB. Such pronounced polarization characteristics are more than an order of magnitude larger than conventional UV-inscribed uniform FBGs in standard fiber and we believe such degrees of polarization-dependency have not been observed in previous studies of PBP inscribed FBGs. As mentioned above, since the number of constituent periods is reduced for the $2^{\text {nd }}$ order grating structure, the grating's second-order Bragg resonance at $\sim 1560.9 \mathrm{~nm}$ is weaker expectedly and the effective birefringence and $\Delta \gamma$ are less pronounced at $\Delta \mathrm{n}_{\text {eff }} \sim 2.9 \times 10^{-5}$ and $1.5 \mathrm{~dB}$ respectively as shown in Fig. $2 \mathrm{~b}$. Note that by changing the fiber scan speed, Bragg resonances can be provided anywhere in the C-band and such distinct birefringence and polarization-dependent reflectivity characteristics are consistently observed.

\section{Conclusion}

High spectral quality FBGs with $1^{\text {st }}$ order Bragg resonances within the C-band are realized using PBP inscription technique. Resolving the fabrication limitations imposed by the fiber curvature has enabled tightly-confined index modification using low inscription energy, thereby creating high index modulation, sub- $\mu \mathrm{m}$ period grating structures. The achieved FBGs exhibit high degrees of polarization-dependent spectral responses which are potentially useful for polarization-selective resonator devices. Detail analysis of the grating polarization characteristics with different grating order and focusing conditions will be reported in near future.

\section{References}

[1] N. Jovanovic et al, "Stable high-power continuous-wave Yb3+-doped silica fiber laser utilizing a point-by-point inscribed fiber Bragg grating", Opt. Lett., 32, 1486-1489, 2007.

[2] A. Martinez et al, "Direct writing of fiber Bragg gratings by femtosecond laser", Electron. Lett., 40, 1170-1172, 2004.

[3] Y. Lai et al., "Microchannels in conventional single-mode fibers", Opt. Lett., 31, 2559-2561, 2006.

[4] Y. Cheng et al, "Control of the cross-sectional shape of a hollow microchannel embedded in photostructurable glass by use of a femtosecond laser", Opt. Lett., 28, 55-57, 2003. 